JOURNAL OF THE

AMERICAN MATHEMATICAL SOCIETY

Volume 18, Number 3, Pages 711-734

S 0894-0347(05)00483-2

Article electronically published on March 24, 2005

\title{
MINKOWSKI'S CONJECTURE, WELL-ROUNDED LATTICES AND TOPOLOGICAL DIMENSION
}

\author{
CURTIS T. MCMULLEN
}

\section{Contents}

1. Introduction

2. Topology of the torus

3. Lattices and number fields

4. Proof for compact orbits

5. Topology of $\mathbb{R}^{n}$

6. Incompressible subspaces

7. Proof for bounded orbits

8. Minkowski's conjecture

References

\section{INTRODUCTION}

Let $A \subset \mathrm{SL}_{n}(\mathbb{R})$ be the diagonal subgroup, and identify $\mathrm{SL}_{n}(\mathbb{R}) / \mathrm{SL}_{n}(\mathbb{Z})$ with the space of unimodular lattices in $\mathbb{R}^{n}$. In this paper we show that the closure of any bounded orbit

$$
A \cdot L \subset \mathrm{SL}_{n}(\mathbb{R}) / \mathrm{SL}_{n}(\mathbb{Z})
$$

meets the set of well-rounded lattices. This assertion implies Minkowski's conjecture for $n=6$ and yields bounds for the density of algebraic integers in totally real sextic fields.

The proof is based on the theory of topological dimension, as reflected in the combinatorics of open covers of $\mathbb{R}^{n}$ and $T^{n}$.

Minkowski's conjecture. Let $|x|$ and $N(x)$ denote the Euclidean length and norm functions on $\mathbb{R}^{n}$, given by

$$
|x|^{2}=\left|x_{1}\right|^{2}+\cdots+\left|x_{n}\right|^{2} \text { and } N(x)=\left|x_{1} \cdot x_{2} \cdots x_{n}\right| .
$$

Note that $N(x)$ is preserved by the action of the diagonal subgroup

$$
A=\left\{\left(\begin{array}{ccc}
a_{1} & & \\
& \ddots & \\
& & a_{n}
\end{array}\right): a_{i}>0, a_{1} a_{2} \cdots a_{n}=1\right\} \subset \operatorname{SL}_{n}(\mathbb{R}) .
$$

Received by the editors August 27, 2004.

2000 Mathematics Subject Classification. Primary 11H31; Secondary 11E57, 11J83, 55M10, $55 \mathrm{~N} 30$.

Research partially supported by the NSF and the Guggenheim Foundation.

(C)2005 American Mathematical Society Reverts to public domain 28 years from publication 
The following conjecture is due to Minkowski [Ko, p. 18]:

Conjecture 1.1. For any unimodular lattice $L \subset \mathbb{R}^{n}$, we have

$$
\sup _{x \in \mathbb{R}^{n}} \inf _{y \in L} N(x-y) \leq 2^{-n} .
$$

Equality holds if and only if $L=a \cdot \mathbb{Z}^{n}$ for some $a \in A$.

This conjecture is known for $n=2,3,4$ and 5 by work of Minkowski, Remak, Dyson and Skubenko, respectively, [Min, §11], [Re], Dy], [Sk2], [Sk1]. Motivated by the study of algebraic integers, its number-theoretic consequences include:

Conjecture 1.2. Let $K$ be a totally real number field over $\mathbb{Q}$, of degree $n$ and discriminant $D_{K}$. Then for every $x \in K$ there exists an algebraic integer $y \in \mathcal{O}_{K}$ such that

$$
\left|N_{\mathbb{Q}}^{K}(x-y)\right| \leq 2^{-n} \sqrt{D_{K}}
$$

Well-rounded lattices. Given a lattice $L \subset \mathbb{R}^{n}$, let $|L|$ and $N(L)$ denote the infimum of $|y|$ and $N(y)$, respectively, over all nonzero $y \in L$. A vector $y \in L$ is minimal if $|y|=|L|$, and $L$ is well-rounded if its minimal vectors span $\mathbb{R}^{n}$.

By the inequality between the arithmetic and geometric means, we have

$$
N(x)^{1 / n} \leq|x| / \sqrt{n}
$$

for all $x \in \mathbb{R}^{n}$. Therefore, to prove Minkowski's conjecture for a given value of $n$, it suffices to establish the following.

$\left(W_{n}\right)$ For any lattice $L \subset \mathbb{R}^{n}$, there exists an $a \in A$ such that $a \cdot L$ is well-rounded.

$\left(C_{n}\right)$ The covering radius of any well-rounded unimodular lattice satisfies

$$
\sup _{x \in \mathbb{R}^{n}} \inf _{y \in L}|x-y| \leq \sqrt{n} / 2 .
$$

Equality holds if and only if $L=g \cdot \mathbb{Z}^{n}$ for some $g \in \mathrm{SO}_{n}(\mathbb{R})$.

This strategy is used in the proofs cited above.

Compact orbits. In 1956, Birch and Swinnerton-Dyer showed that any counterexample to Minkowski's conjecture with minimal dimension must satisfy $N(L)>$ 0 BiS]; equivalently, $A \cdot L$ must have compact closure in the space of lattices $\mathrm{SL}_{n}(\mathbb{R}) / \mathrm{SL}_{n}(\mathbb{Z})$.

In this paper we will show

Theorem 1.3. If the orbit closure

$$
\overline{A \cdot L} \subset \mathrm{SL}_{n}(\mathbb{R}) / \mathrm{SL}_{n}(\mathbb{Z})
$$

of a lattice $L \subset \mathbb{R}^{n}$ is compact, then it meets the locus of well-rounded lattices.

Corollary 1.4. If the covering conjectures $\left(C_{k}\right)$ hold for all $k \leq n$, then Minkowski's conjecture is also true for all lattices in $\mathbb{R}^{k}, k \leq n$.

Since the covering conjectures are known for $k \leq 6$ [Wd, we obtain

Corollary 1.5. Minkowski's conjecture holds for $n=6$. 
Topology of the torus. The proof of Theorem 1.3 is based on the following topological result.

Theorem 1.6. There is no open covering $T^{k}=U_{1} \cup \cdots \cup U_{k}$ of the $k$-torus such that the map

$$
H_{1}(V, \mathbb{Z}) \rightarrow H_{1}\left(T^{k}, \mathbb{Z}\right)
$$

has rank at most $(i-1)$ for every component $V$ of $U_{i}$.

In the special case where every map $H_{1}(V, \mathbb{Z}) \rightarrow H_{1}\left(T^{k}, \mathbb{Z}\right)$ has rank 0 , this theorem follows from Lebesgue's characterization of the topological dimension of $\mathbb{R}^{k}$.

To apply the result above to lattices, assume for simplicity that $A \cdot L$ is not just bounded but actually compact. Then the following hold.

- The stabilizer $A_{L}$ of $L$ in $A$ is isomorphic to $\mathbb{Z}^{n-1}$.

- $A / A_{L}$ is diffeomorphic to a torus of dimension $k=(n-1)$.

- $L$ arises from a totally real number field via a standard construction (\$3).

For each $a \in A / A_{L}$, there is a unique subspace $M(a) \subset \mathbb{R}^{n}$ spanned by the minimal vectors of the lattice $a \cdot L$. If $\operatorname{dim} M(a)=n$ for some $a$, then $a \cdot L$ is well-rounded and we are done.

Otherwise, the torus $T^{k}=A / A_{L}$ is covered by sets $S_{1}, \ldots, S_{k}$, such that $\operatorname{dim} M(a)=i$ for all $a \in S_{i}$. The subspace $M(a)$ varies continuously over $S_{i}$, yielding a monodromy map

$$
\pi_{1}\left(S_{i}, a\right) \rightarrow \mathrm{SL}(M(a)) .
$$

The monodromy acts by diagonal matrices, so the image of (1.2) is an abelian group no larger than $\mathbb{Z}^{i-1}$.

On the other hand, the natural map $\pi_{1}\left(S_{i}, *\right) \rightarrow \pi_{1}\left(T^{k}\right)$ has the same kernel as (1.2), so its rank is also at most $i-1$. By thickening the sets $S_{i}$ slightly, we obtain a covering of $T^{k}$ by open sets $U_{1}, \ldots, U_{k}$ whose existence is ruled out by Theorem 1.6. This completes the proof of Theorem 1.3 in the case where $A \cdot L$ is compact.

To handle the general case, we extend Theorem 1.6 to open coverings of $\mathbb{R}^{k}$, using the Čech-deRham complex of bounded differential forms (\$5). We also study those subspaces $M \subset \mathbb{R}^{n}$ which satisfying $\inf _{A}\|\operatorname{det}(a \cdot M)\|>0(\Phi 6)$. These discussions reveal that many features of the case where $A \cdot L$ is compact carry over to the case where $A \cdot L$ is only bounded, allowing us to deduce Theorem 1.3 and Corollary 1.4 in $\$ 7$ and $₫ 8$

The case $n=2$. To give a picture of the theorem's underlying geometry, we sketch a proof that $A \cdot L$ meets the well-rounded locus whenever $L$ is a lattice in the plane.

Recall that the set of 2-dimensional lattices can be parameterized by the upper halfplane $\mathbb{H} \subset \mathbb{C}$, via the correspondence

$$
\tau \mapsto L_{\tau}=\mathbb{Z} \oplus \mathbb{Z} \tau .
$$

The lattice $L_{\tau}$ is well-rounded if and only if $\tau$ belongs to $W=\mathrm{SL}_{2}(\mathbb{Z}) \cdot C$, where $C$ is the circular $\operatorname{arc} \exp (2 \pi i[\pi / 3, \pi / 2]) \subset \mathbb{C}$. The locus $W$ is a spine for the action of $\mathrm{SL}_{2}(\mathbb{Z})$ on $\mathbb{H}$; it is a tree connecting the fixed-points of the elliptic elements in $\mathrm{SL}_{2}(\mathbb{Z})$, and each of its complementary regions is contained in a horoball (see Figure (1). 


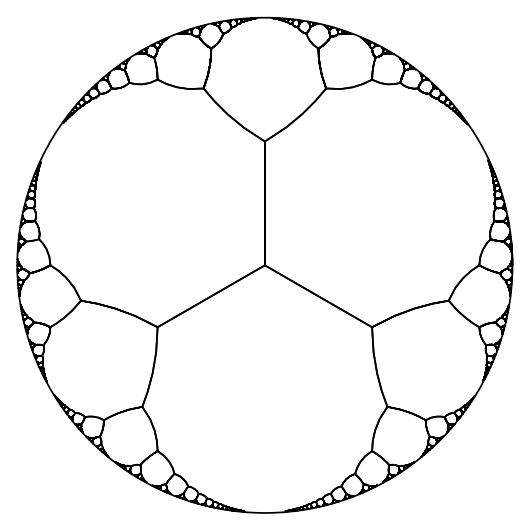

Figure 1. The well-rounded spine for $\mathrm{SL}_{2}(\mathbb{Z})$.

Now it is easy to check that the orbit $A \cdot L_{\tau}$ of a given lattice is simply a hyperbolic geodesic through $\tau \in \mathbb{H}$. Since no complete geodesic can be entirely contained in a horoball, $A \cdot L_{\tau}$ must meet $W$, and therefore $a \cdot L$ is well-rounded for some $a \in A$.

The well-rounded lattices in $\mathbb{R}^{n}$ also form a compact spine for the space

$$
\mathrm{SO}_{n}(\mathbb{R}) \backslash \mathrm{SL}_{n}(\mathbb{R}) / \mathrm{SL}_{n}(\mathbb{Z})
$$

and have been used to study its cohomology [So], Ash], AM]. It would be interesting to have a more complete description of the way geodesic flats can meet the well-rounded spine in higher dimensions.

Notes and references. The torus covering Theorem 1.6 above aims to isolate the topological core of Minkowski's conjecture. Intricate topological and combinatorial arguments also appear in the proofs for $n=3,4,5$ by Remak, Dyson and Skubenko.

For a short proof of Minkowski's conjecture for $n=3$, see Da. A simplified treatment of the case $n=5$, using the result of Birch and Swinnerton-Dyer cited above, is given by Bambah and Woods in [BW]. Related problems and results are discussed in GL, Chapter 7].

I would like to thank G. Nebe for introducing me to Minkowski's conjecture, in connection with her paper $[\mathrm{BN}]$ with E. Bayer-Fluckiger.

Notation. The open ball of radius $R$ about $p \in \mathbb{R}^{n}$ will be denoted by $B^{n}(p, R)$. We will also use the abbreviations $B^{n}(R)=B^{n}(0, R), S^{n-1}(R)=\partial B^{n}(R), B^{n}=$ $B^{n}(1)$ and $S^{n-1}=S^{n-1}(1)$. We let $\operatorname{diag}\left(a_{1}, \ldots, a_{n}\right)$ denote a matrix in $A$ specified by its diagonal entries.

\section{TOPOLOGY OF THE TORUS}

Let $\mathfrak{U}$ be an open covering of a topological space. The order of $\mathfrak{U}$ is the greatest integer $n$ such that some $(n+1)$ distinct elements of $\mathfrak{U}$ have nonempty intersection. (Equivalently, the order is the dimension of the nerve of $\mathfrak{U}$.)

One can also consider the homology of multiple intersections. In this section we will establish

Theorem 2.1. Let $\mathfrak{U}$ be an open covering of the torus $T^{n}=\mathbb{R}^{n} / \mathbb{Z}^{n}$. Suppose that for any component $V$ of the intersection $U_{1} \cap U_{2} \cap \cdots \cap U_{k}$ of $1 \leq k \leq n$ distinct 
elements of $\mathfrak{U}$, the image of the map

$$
H_{1}(V, \mathbb{Z}) \rightarrow H_{1}\left(T^{n}, \mathbb{Z}\right)
$$

has rank at most $(n-k)$. Then $\mathfrak{U}$ has order at least $n$.

Corollary 2.2. There is no open covering $T^{n}=U_{1} \cup \cdots \cup U_{n}$ such that the map

$$
H_{1}(V, \mathbb{Z}) \rightarrow H_{1}\left(T^{n}, \mathbb{Z}\right)
$$

has rank at most $(i-1)$ for every component $V$ of $U_{i}$.

Proof. Apply the theorem above to the covering $\mathfrak{U}=\left\{U_{1}, \ldots, U_{n}\right\}$.

Dimension and cohomology. We begin by sketching the proof of Theorem 2.1 in two special cases.

(1) First suppose every intersection $U_{1} \cap \cdots \cap U_{k}$ is contractible. Then the cohomology $H^{*}\left(T^{n}, \mathbb{Z}\right)$ agrees with Čech cohomology $H^{*}(\mathfrak{U}, \mathbb{Z})[\mathrm{BT}$, Thm. 15.8]. Since $H^{n}(\mathfrak{U}, \mathbb{Z}) \cong H^{n}\left(T^{n}, \mathbb{Z}\right) \cong \mathbb{Z}$, the covering $\mathfrak{U}$ must have order at least $n$.

(2) Now suppose just that $H_{1}(U, \mathbb{Z})$ maps to zero in $H_{1}\left(T^{n}, \mathbb{Z}\right)$, for every $U \in$ $\mathfrak{U}$. Since $T^{n}$ is compact, we can slightly shrink the elements $U \in \mathfrak{U}$ so they become subcomplexes of a finite triangulation of $T^{n}$. Then by our homological assumption, each $U \in \mathfrak{U}$ lifts to a bounded open set under the universal covering map $\pi: \mathbb{R}^{n} \rightarrow T^{n}$. The components of $\left(\pi^{-1}(U), U \in\right.$ $\mathfrak{U})$ therefore give a covering $\mathfrak{V}$ of $\mathbb{R}^{n}$ by open sets of uniformly bounded diameter. By Lebesgue's characterization of the topological dimension of $\mathbb{R}^{n}[\mathrm{HW}$ Thms. IV.2, V 8], $\mathfrak{V}$ has order at least $n$, so the same is true of $\mathfrak{U}$.

Examples. Theorem 2.1, unlike the special cases just sketched, also yields information when elements of $\mathfrak{U}$ are homologically nontrivial. For example, if $T^{2}$ is covered by a pair of open annuli $U_{1}, U_{2}$, it implies $U_{1} \cap U_{2}$ must also contain an essential annulus. Similarly, Corollary 2.2 implies the 3 -torus admits no covering

$$
T^{3}=U_{1} \cup U_{2} \cup U_{3}
$$

such that the components of $U_{1}$ are homeomorphic to balls and the components of $U_{2}$ and $U_{3}$ are homeomorphic to solid tori $\left(B^{2} \times S^{1}\right)$.

We remark that the hypotheses of Theorem 2.1]imply the map

$$
H_{n-k-1}\left(U_{1} \cap \cdots \cap U_{k}, \mathbb{Z}\right) \rightarrow H_{n-k-1}\left(T^{n}, \mathbb{Z}\right)
$$

is zero for $1 \leq k \leq n$. This condition, however, is too weak to control the order of $\mathfrak{U}$; for example, it holds for the covering $T^{n}=U_{1} \cup U_{2}$ where $U_{1}=T^{n}-\{p\}$ and $U_{2}$ is a ball about $p$.

The Čch-deRham complex. To set the stage for the proof of Theorem 2.1, we recall the definition of the $\breve{C} e c h$-deRham complex $C^{*}\left(\mathfrak{U}, \Omega^{*}\right)($ see $[\mathrm{BT}, \S 8])$.

Let $\mathfrak{U}=\left\{U_{i}\right\}$ be an open covering of a smooth manifold $M$. For any set of distinct indices $I=\left\{i_{1}, \ldots, i_{p}\right\}$, let $U_{I}=U_{i_{1}} \cap U_{i_{2}} \cap \cdots \cap U_{i_{p}}$. Let $\Omega^{q}$ denote the sheaf of smooth $q$-forms on $T^{n}$, and let

$$
C^{p}\left(\mathfrak{U}, \Omega^{q}\right)=\prod_{|I|=p+1} \Omega^{q}\left(U_{I}\right)
$$




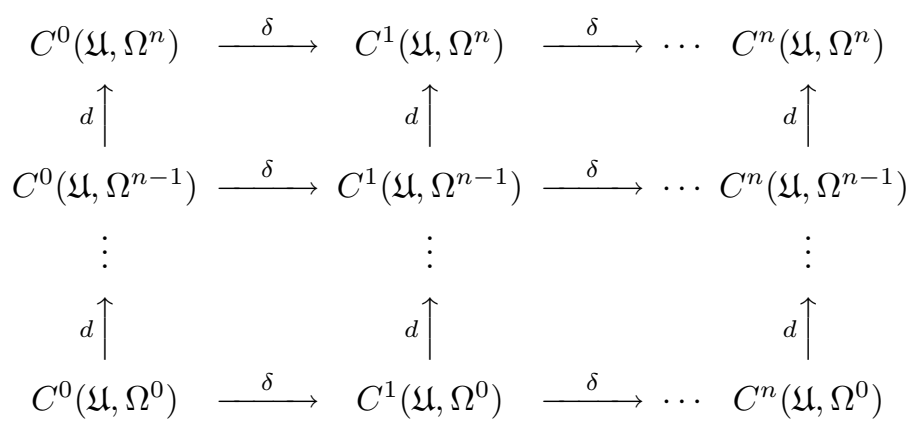

Figure 2. The Čech-deRham complex.

be the space of Čech $p$-chains with values in $\Omega^{q}$. The double complex $C^{*}\left(\mathfrak{U}, \Omega^{*}\right)$ comes equipped with a deRham differential $d$ and a Čech differential $\delta$, satisfying $d^{2}=\delta^{2}=0$ (Figure 2). The diagonal complex

$$
A^{r}=\bigoplus_{p+q=r} C^{p}\left(\mathfrak{U}, \Omega^{q}\right)
$$

then admits a differential $D=d+(-1)^{q} \delta$ satisfying $D^{2}=0$.

Theorem 2.3. The cohomology groups $H_{D}^{r}\left(C^{*}\left(\mathfrak{U}, \Omega^{*}\right)\right)$ of the diagonal complex are isomorphic to the deRham cohomology groups $H_{D R}^{r}(M)$.

This result, which can be regarded as a generalization of the Mayer-Vietoris theorem, follows easily from the fact that the horizontal rows in Figure 2 are exact sequences; see [BT, Prop. 8.8].

Covering spaces. Now suppose $M$ is connected, with universal cover $\widetilde{M} \rightarrow M$. Then $\pi_{1}(M)$ acts on the vector space $\Omega^{q}(\widetilde{M})$, making it into a flat vector bundle over $M$.

Let $\mathcal{F}^{q}$ denote the sheaf of flat sections of this bundle. Then for any connected open set $U \neq \emptyset$ in $M$ we have

$$
\mathcal{F}^{q}(U) \cong \Omega^{q}\left(M_{U}\right)
$$

where $M_{U}=\widetilde{M} / \pi_{1}(U) \rightarrow M$ is the covering space of $M$ determined by $\pi_{1}(U)$. The natural lifting $s: U \rightarrow M_{U}$ induces a restriction map $\mathcal{F}^{q}(U) \rightarrow \Omega^{q}(U)$, and thus an element $\omega \in \mathcal{F}^{q}(U)$ can be regarded as a form on $U$ equipped with an extension to $M_{U}$.

The restriction map $\mathcal{F}^{q} \rightarrow \Omega^{q}$ respects both the Čech and deRham differentials, so it determines a chain map

$$
C^{p}\left(\mathfrak{U}, \mathcal{F}^{q}\right) \rightarrow C^{p}\left(\mathfrak{U}, \Omega^{q}\right) .
$$

Using the differential $D=d+(-1)^{q} \delta$ on both sides, we then get a map between the cohomology groups of the corresponding diagonal complexes.

Theorem 2.4. The natural map $H_{D}^{r}\left(C^{*}\left(\mathfrak{U}, \mathcal{F}^{*}\right)\right) \rightarrow H_{D}^{r}\left(C^{*}\left(\mathfrak{U}, \Omega^{*}\right)\right)$ is a surjection for every $r$. 
Proof. By Theorem 2.3 every class $x \in H_{D}^{r}\left(C^{*}\left(\mathfrak{U}, \Omega^{*}\right)\right)$ is represented by a closed form $\omega \in \Omega^{r}(M)$. Lifting this form to the universal cover, we obtain a cocycle $y=\left(\widetilde{\omega} \mid U_{i}\right) \in C^{0}\left(\mathfrak{U}, \mathcal{F}^{r}\right)$ mapping to $x$.

Proof of Theorem 2.1. Let $\mathfrak{U}$ be a covering of $T^{n}$ satisfying the hypotheses of the theorem.

We claim the sequence

$$
C^{p}\left(\mathfrak{U}, \mathcal{F}^{q-1}\right) \stackrel{d}{\rightarrow} C^{p}\left(\mathfrak{U}, \mathcal{F}^{q}\right) \stackrel{d}{\rightarrow} C^{p}\left(\mathfrak{U}, \mathcal{F}^{q+1}\right)
$$

is exact, for any $p, q \geq 0$ satisfying $p+q=n$. To see this, suppose $\left(\omega_{I} \in\right.$ $\left.\mathcal{F}^{q}\left(U_{I}\right),|I|=p+1\right)$ is an element of the central term above, with $d \omega_{I}=0$ for all $I$. Let $V$ be a component of $U_{I}$. Then by hypothesis, the image of $H_{1}(V, \mathbb{Z})$ in $H_{1}(M, \mathbb{Z})$ has rank at most $n-(p+1)=q-1$. Thus the covering space $M_{V} \rightarrow M$ determined by $\pi_{1}(V)$ is isomorphic to $T^{k} \times \mathbb{R}^{n-k}$ with $k<q$. In particular, we have

$$
H_{D R}^{q}\left(M_{V}\right) \cong H_{D R}^{q}\left(T^{k} \times \mathbb{R}^{n-k}\right)=0
$$

so any closed form $\omega \in \Omega^{q}\left(M_{V}\right) \cong \mathcal{F}^{q}(V)$ is exact. Applying this fact to each component of $U_{I}$, we obtain a form $\eta_{I} \in \mathcal{F}^{q-1}\left(U_{I}\right)$ satisfying $d \eta_{I}=\omega_{I}$; therefore the sequence (2.1) is also exact.

Now assume $\mathfrak{U}$ has order less than $n$. We claim that $H_{D}^{n}\left(C^{*}\left(\mathfrak{U}, \mathcal{F}^{*}\right)\right)=0$. Indeed, let $[y]$ be an $n$-dimensional cohomology class, represented by $y=\sum_{0}^{n} y_{p}$ with $y_{p} \in$ $C^{p}\left(\mathfrak{U}, \mathcal{F}^{n-p}\right)$. Since $D y=0$, we have $d y_{0}=0$, and therefore $y_{0}=d z_{0}$ by exactness of (2.1). Thus after replacing $y$ with $y-D z_{0}$ (which does not change its cohomology class), we can assume $y_{0}=0$ (see the following diagram).

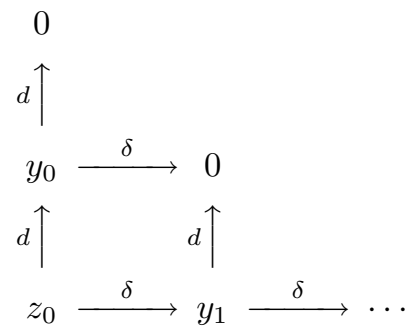

Once $y_{0}=0$, we also have $d y_{1}=0$, and therefore we can similarly modify $y$ by a coboundary $D z_{1}$ to make $y_{1}=0$. Continuing in this way, we ultimately obtain a cycle $y_{n} \in C^{n}\left(\mathfrak{U}, \mathcal{F}^{0}\right)$ representing the cohomology class $[y]$. But if the order of $\mathfrak{U}$ is less than $n$, then $U_{I}$ is empty whenever $|I|=n+1$; thus $y_{n}=0$, and therefore $H_{D}^{n}\left(C^{*}\left(\mathfrak{U}, \mathcal{F}^{*}\right)\right)=0$.

On the other hand, the preceding theorems show that $H_{D}^{n}\left(C^{*}\left(\mathfrak{U}, \mathcal{F}^{*}\right)\right)$ maps surjectively to $H_{D R}^{n}\left(T^{n}\right)=\mathbb{R}$; therefore $\mathfrak{U}$ has order greater than or equal to $n$.

Remark. By passing from $\Omega^{p}$ to $\mathcal{F}^{p}$, we keep track of the fact that all the forms arising in the proof are defined not just on the intersections $U_{I}$, but also on suitable covering spaces of $T^{n}$. 


\section{LATtiCES AND NUMBER FIELDS}

In this section we review the construction of lattices using totally real fields, and we show

Theorem 3.1. For any unimodular lattice $L \subset \mathbb{R}^{n}$, the following conditions are equivalent:

(1) $A \cdot L$ is compact.

(2) $L$ arises from a full module $M$ in a totally real field $K / \mathbb{Q}$.

(3) We have $N(L)>0$, and $\{N(y): y \in L\}$ is a discrete subset of $\mathbb{R}$.

Lattices. A lattice is a discrete subgroup $L \subset \mathbb{R}^{n}$ isomorphic to $\mathbb{Z}^{n}$; it is unimodular if $|\operatorname{det}(L)|=\operatorname{vol}\left(\mathbb{R}^{n} / L\right)=1$. The space of all unimodular lattices can be identified with the homogeneous space

$$
G / \Gamma=\mathrm{SL}_{n}(\mathbb{R}) / \mathrm{SL}_{n}(\mathbb{Z})
$$

via the bijection $[g] \mapsto g \cdot \mathbb{Z}^{n}=L$.

Compactness. We define $|L|$ and $N(L)$ to be infimum of $|y|$ and $N(y)$, respectively, over nonzero elements $y \in L$. By Mahler's criterion [Rag, 10.9],

$$
\bar{E} \subset \mathrm{SL}_{n}(\mathbb{R}) / \mathrm{SL}_{n}(\mathbb{Z}) \text { is compact } \Longleftrightarrow \inf \{|L|: L \in E\}>0 .
$$

Similarly, since $N(y)^{1 / n} \leq|y| / \sqrt{n}$, we can assert that

$$
\overline{A \cdot L} \text { is compact } \Longleftrightarrow N(L)>0 .
$$

The function $N(L)$ is also sometimes denoted $m_{H}(L)$ [BiS], [BW].

Compact orbits. Number fields can be used to give concrete examples of lattices such that $A \cdot L$ is compact.

Let $K$ be a totally real number field of degree $n$ and discriminant $D_{K}$ over $\mathbb{Q}$. The $n$ distinct embeddings of $K$ into $\mathbb{R}$ give an embedding

$$
v: K \hookrightarrow \mathbb{R}^{n}
$$

sending the ring of integers $\mathcal{O}_{K}$ to a lattice of determinant $\sqrt{D_{K}}$. More generally, if $M \subset K$ is a full module, i.e., an additive subgroup isomorphic to $\mathbb{Z}^{n}$, then

$$
L=\alpha \cdot v(M) \subset \mathbb{R}^{n}
$$

is a unimodular lattice, where $\alpha=|\operatorname{det} v(M)|^{-1 / n}$.

The module $M$ is invariant under the order

$$
\mathfrak{o}=\{x \in K: x M \subset M\}
$$

(a subring of finite index in $\mathcal{O}_{K}$ ), and hence $L$ is invariant under the subgroup $A_{L} \subset A$ corresponding to the totally positive units

$$
\mathfrak{o}_{+}^{*}=\mathfrak{o}^{*} \cap v^{-1}\left(\mathbb{R}_{+}^{n}\right) .
$$

By Dirichlet's theorem [BoS, Ch. 2, §4.3], the unit group $\mathfrak{o}_{+}^{*}$ is isomorphic to $\mathbb{Z}^{n-1}$. Thus $A / A_{L}$ is a compact torus, so $A \cdot L \subset \mathrm{SL}_{n}(\mathbb{R}) / \mathrm{SL}_{n}(\mathbb{Z})$ is compact as well.

Note that by replacing $M$ with $x M$ for suitable $x \in K$, we can always reduce to the case where $M$ is an ideal in $\mathfrak{o}$. Then we have

$$
N(v(x))=\left|N_{\mathbb{Q}}^{K}(x)\right| \in \mathbb{Z}
$$

for all $x \in M$. Consequently $\{N(y): y \in L\}$ is discrete and $N(L)>0$. 
Proof of Theorem 3.1. (1) $\Longrightarrow(2)$. Suppose $A \cdot L$ is compact, and let $A_{L}$ denote the stabilizer of $L$ in $A$. Then $L \otimes \mathbb{Q}$ is a module over the commutative algebra $K=\mathbb{Q}\left[A_{L}\right] \subset M_{n}(\mathbb{R})$. The matrices in $A$ have only real eigenvalues, so $K$ is a direct sum of $m$ totally real fields, and therefore the rank of $\mathfrak{o}_{K}^{*}$ is $n-m$. But the matrix group $A_{L} \cong \mathbb{Z}^{n-1}$ embeds in the unit group $\mathfrak{o}_{K}^{*}$, so $m=1$ and $K$ itself is a totally real field. Thus $L \otimes \mathbb{Q}$ is a 1-dimensional vector space over $K$, so the lattice $L$ itself is obtained from a full module $M \subset K$ by the construction above.

The implication $(2) \Longrightarrow(3)$ is immediate from discreteness of the norm $N_{\mathbb{Q}}^{K}(x)$ on $M$.

To see $(3) \Longrightarrow(1)$, observe that the map $g \mapsto N(g x)=\phi(x)$ gives a proper embedding of $A \backslash G$ into the space of degree $n$ polynomials on $\mathbb{R}^{n}$. There is a finite set $E \subset \mathbb{Z}^{n}$ such that $\phi \mid E$ determines $\phi$. Consequently, if the values of $N(x)$ on $L=g \cdot \mathbb{Z}^{n}$ are discrete, then $[g] \cdot \Gamma$ is closed in $A \backslash G$, and therefore $A \cdot L$ is closed in $G / \Gamma$. Since $N(L)>0$, by (3.1) the orbit $A \cdot L$ is actually compact.

Notes and references. For more on compact $A$-orbits, minimal sets and totally real fields, see $[\mathrm{LW}]$ and $[\mathrm{Oh}]$. Lattices such that $A \cdot L$ is closed are characterized in TW]. Further references for number-theoretic constructions of lattices include $\mathrm{CoS}$, [Ba1], $\mathrm{Ba} 2$ and $\mathrm{BN}$.

We remark that Margulis has proposed

Conjecture 3.2. For $n \geq 3, \overline{A \cdot L} \subset \mathrm{SL}_{n}(\mathbb{R}) / \mathrm{SL}_{n}(\mathbb{Z})$ is compact if and only if $A \cdot L$ is compact.

See $[\mathrm{Mg}$, Conj. 9]. This conjecture is equivalent to the assertion that any lattice with $N(L)>0$ arises from a number field. The case $n=3$ implies Littlewood's conjecture, that $\lim \inf n \cdot\|n \alpha\| \cdot\|n \beta\|=0$ for all real numbers $\alpha, \beta$. Related material appears in the paper [CaS] on products of three linear forms, dating from the same period as the work [BiS] on Minkowski's conjecture.

\section{Proof For COMPACt orbits}

In this section we prove

Theorem 4.1. Let $L \subset \mathbb{R}^{n}$ be a lattice such that $A \cdot L$ is compact in $\mathrm{SL}_{n}(\mathbb{R}) / \mathrm{SL}_{n}(\mathbb{Z})$. Then $a \cdot L$ is well-rounded for some $a \in A$.

This case is sufficient for applications to number fields, and the framework of its proof provides a guide to the general case (where $A \cdot L$ is only assumed to be bounded).

Convex hulls. To organize the minimal vectors of $a \cdot L$ as $a \in A$ varies, we will use a convex hull construction introduced by Skubenko [Sk2]; see also [BW].

We begin with some terminology. Let $B \subset \mathbb{R}^{n}$ be a nonempty, closed, convex set. The relative interior $B^{\circ}$ of $B$ is taken with respect to the smallest hyperplane $S \supset B$; it is always nonempty.

Let $\phi: \mathbb{R}^{n} \rightarrow \mathbb{R}$ be a nonzero linear form, with $\phi \mid B$ bounded below. If $\phi \mid B$ assumes its minimum on a nonempty subset $F \subset B$, then $F$ is a face of $B$, dual to the support function $\phi$. Any point $p \in \partial B$ is contained in a unique open face $F^{\circ}$.

The convex hull of a set $E \subset \mathbb{R}^{n}$, denoted hull $(E)$, is the smallest closed, convex set containing $E$. A convex set is a polyhedron if it is the convex hull of a finite set of points. We say $\partial B$ is a polyhedral complex if every face of $B$ is a convex polyhedron. 


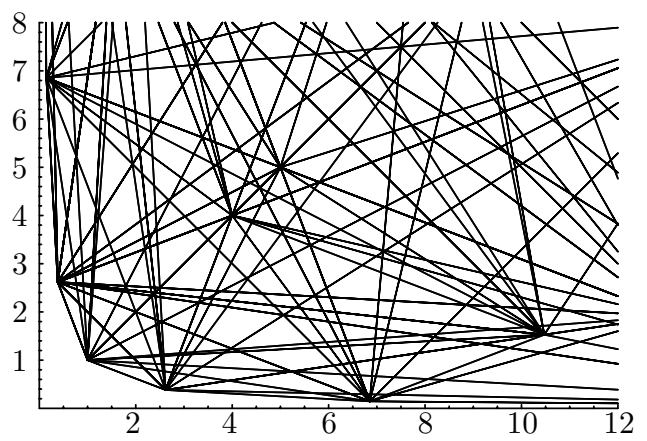

Figure 3. The convex hull of $L^{2}$ in $\mathbb{R}_{+}^{2}$.

The convex hull of $L^{2}$. Now let $L$ be a lattice in $\mathbb{R}^{n}$, and let $a=\operatorname{diag}\left(a_{1}, \ldots, a_{n}\right)$ $\in A$. To find the minimal vectors of $a \cdot L$, consider the convex hull $B$ of

$$
L^{2}=\left\{y^{2}: y \in L, y \neq 0\right\} \subset \mathbb{R}_{+}^{n},
$$

where $y^{2}=\left(y_{1}^{2}, \ldots, y_{n}^{2}\right)$. Define a linear form $\phi_{a}: \mathbb{R}^{n} \rightarrow \mathbb{R}$ by

$$
\phi_{a}(x)=\sum a_{i}^{2} x_{i} .
$$

Then for any $y \in L$, we have $\phi_{a}\left(y^{2}\right)=|a y|^{2}$. This readily implies

Theorem 4.2. The form $\phi_{a} \mid B$ achieves its minimum on the polyhedral face

$$
F=\operatorname{hull}\left(y_{1}^{2}, \ldots, y_{m}^{2}\right)
$$

of $B$, where $a \cdot\left( \pm y_{1}, \ldots, \pm y_{m}\right)$ are the minimal vectors of $a \cdot L$.

Note: the set $\left(y_{1}^{2}, \ldots, y_{m}^{2}\right)=F \cap L^{2}$ includes the extreme points of $F$, but additional points may also appear.

Corollary 4.3. If $L-\{0\}$ is disjoint from the coordinate axes of $\mathbb{R}^{n}$, then $\partial B$ is a polyhedral complex.

Proof. In this case every support function for $B$ is given by $\phi(x)=\lambda \phi_{a}(x)$, for some $a \in A$ and $\lambda>0$.

Compare $[\mathrm{BW}]$.

Example. The locus $B=\operatorname{hull}\left(L^{2}\right)$ for $L=\mathbb{Z}(1,1) \oplus \mathbb{Z}\left(\gamma_{+}, \gamma_{-}\right), \gamma_{ \pm}=(1 \pm \sqrt{5}) / 2$, is shown in Figure 3. The vertices of $\partial B$ are the powers $\left(\epsilon^{n}, \epsilon^{-n}\right)$ of the totally positive fundamental unit $\epsilon=(3+\sqrt{5}) / 2$.

Stratification. Any point $x \in \partial B$ lies in a unique open face $F^{\circ}$, with $F=$ $\operatorname{hull}\left(y_{1}^{2}, \ldots, y_{m}^{2}\right)$ as above. Let

$$
M(x)=\left\langle y_{1}, \ldots, y_{m}\right\rangle \subset L \otimes \mathbb{R}
$$

be the real vector space spanned by the corresponding points of $L$. Then for any support function $\phi_{a}$ that achieves its minimum on $F^{\circ}$, the span of the minimal vectors of $a \cdot L$ is the space $a \cdot M(x)$. 
The level sets of the function $\delta(x)=\operatorname{dim} M(x)$ determine a stratification

$$
\partial B=\bigcup_{1}^{n} \widetilde{S}_{i}, \quad \widetilde{S}_{i}=\{x \in \partial B: \delta(x)=i\} .
$$

Each stratum is a union of open faces of $B$.

Theorem 4.4. For each $i \leq n$, the union $\widetilde{S}_{1} \cup \cdots \cup \widetilde{S}_{i}$ is closed.

Proof. The closure of $\widetilde{S}_{i}$ is the union of the faces $F$ of $\partial B$ such that $F^{\circ} \subset \widetilde{S}_{i}$. Since we have $\partial F \cap L^{2} \subset F \cap L^{2}$, the lattice points corresponding to any face $G \subset \partial F$ span a subspace of dimension at most $i$.

Theorem 4.5. If $x$ and $y$ belong to the same connected component of $\widetilde{S}_{i}$, then $M(x)=M(y)$.

Proof. Any two points $x$ and $y$ in the same component of $\widetilde{S}_{i}$ are joined by a chain of open faces of $\partial B$. Thus it suffices to treat the case where $x \in F^{\circ}, y \in G^{\circ}$ and $F^{\circ} \cup G^{\circ}$ is connected. But in this case either $F \supset G$ or $G \supset F$, and therefore $M(x) \supset M(y)$ or $M(y) \supset M(x)$. Since $M(x)$ and $M(y)$ have the same dimension, they are equal.

Number field case. Now assume $A \cdot L$ is compact, with stabilizer $A_{L} \subset A$.

By Theorem 3.1, $L$ arises from a full module in the totally real field $K=\mathbb{Q}\left[A_{L}\right] \subset$ $M_{n}(\mathbb{R})$, and we have $N(L)>0$. In particular, $y=0$ is the only point of $L$ lying on a coordinate axis, and therefore $\partial B=\partial \operatorname{hull}\left(L^{2}\right)$ is a polyhedral complex. The natural action of $A_{L}$ on $L^{2}$ by $y^{2} \mapsto(a y)^{2}=a^{2} y^{2}$ extends to a linear action on $\mathbb{R}_{+}^{n}$, preserving $B$.

Theorem 4.6. The quotient space $S=\partial B / A_{L}$ is homeomorphic to the torus $T^{n-1}$, with $\partial B \cong \mathbb{R}^{n-1}$ as its universal cover.

Proof. The map $f(x)=x / N(x)$ sends $B$ homeomorphically to the hyperboloid $H \subset \mathbb{R}_{+}^{n}$ defined by $N(x)=1$ and respects the action of $A_{L}$. Clearly $A \cong \mathbb{R}^{n-1}$ acts simply transitively on $H$, so we have $\partial B / A_{L} \cong H / A_{L} \cong A / A_{L} \cong T^{n-1}$.

Monodromy over a stratum. Note that $M(a x)=a \cdot M(x)$ for any $a \in A_{L}$ and $x \in \partial B$. Thus the stratification $\partial B=\bigcup \widetilde{S}_{i}$ is invariant under the action of $A_{L}$ and descends to give a partition of $S$ into strata $S_{i}=\widetilde{S}_{i} / A_{L}$. Each $S_{i}$ is a finite union of open polyhedral cells. Similarly, $\delta(x)$ descends to a function on $S$ with level sets $S_{i}$.

Our next result controls the topological complexity of a stratum. Given a subspace $M \subset \mathbb{R}^{n}$, let

$$
A_{M, L}=\left\{a \in A_{L}: a \cdot M=M\right\} .
$$

Theorem 4.7. If $\operatorname{dim}(M)=r>0$, then the free abelian group $A_{M, L}$ has rank at most $\operatorname{gcd}(r, n)-1$.

Proof. The rational vector spaces $L_{\mathbb{Q}}=L \otimes \mathbb{Q}$ and $M_{\mathbb{Q}}=(L \cap M) \otimes \mathbb{Q}$ are also vector spaces over the field $k=\mathbb{Q}\left[A_{M, L}\right] \subset K$. Thus $s=\operatorname{deg}(k / \mathbb{Q})$ divides both $n=\operatorname{dim} L_{\mathbb{Q}}$ and $r=\operatorname{dim} M_{\mathbb{Q}}$. Since $A_{M, L}$ embeds into the group of units $\mathcal{O}_{k}^{*}$, its rank is at most $s-1$. 
Corollary 4.8. For any $x \in S_{i}$, the image of the natural map

$$
\rho: \pi_{1}\left(S_{i}, x\right) \rightarrow \pi_{1}(S)
$$

is an abelian group of rank at most $\operatorname{gcd}(i, n)-1$.

Proof. Choose a point $y \in \widetilde{S}_{i}$ lying over $x \in S_{i}$. Given $g \in \pi_{1}\left(S_{i}, x\right)$, let $a=\rho(g) \in$ $A_{L} \cong \pi_{1}(S)$. Then $z=a \cdot y$ lies in the same component of $\widetilde{S}_{i}$ as $y$, as can be seen by lifting a path representative of $[g]$. This implies that

$$
M(y)=M(z)=M(a \cdot y)=a \cdot M(y),
$$

and therefore $a \in A_{M(y), L}$. Consequently the rank of the image of $\rho$ is bounded by the rank of $A_{M(y), L}$, which is in turn bounded by $\operatorname{gcd}(i, n)-1$, since $\operatorname{dim} M(y)=$ $\delta(x)=i$.

Theorem 4.9. Every stratum $S_{i}$ of $S$ is the deformation retract of an open set $U_{i} \subset S$.

Proof. After refining the polyhedral decomposition of $S$ to a triangulation, we can regard $S$ as a finite simplicial complex and $S_{i}$ as an open subset of the closed subcomplex $T=S_{1} \cup \cdots \cup S_{i}$. Using barycentric subdivision, one can construct an open neighborhood $V$ of $T$ which admits a strong deformation retraction to $T$ [EM, $§ I I .9]$. This means there is a homotopy $\rho: V \times I \rightarrow V$ such that $\rho_{t} \mid T=$ id for all $t, \rho_{0} \mid V=$ id and $\rho_{1}(V)=T$. Letting $U_{i}=\rho_{1}^{-1}\left(S_{i}\right)$, we obtain an open subset of $S$ that deforms to $S_{i}$ under the homotopy $\rho_{t} \mid U_{i}$.

Proof of Theorem 4.1. The preceding result provides a covering of $S \cong T^{n-1}$ by open sets $U_{i}$ that deformation retract to the strata $S_{i}$. Then for any component $V$ of $U_{i}$, the map

$$
H_{1}(V, \mathbb{Z}) \rightarrow H_{1}(S, \mathbb{Z})
$$

has rank at most $\operatorname{gcd}(n, i)-1 \leq i-1$, since it has the same image as the map

$$
\pi_{1}(V, x) \cong \pi_{1}\left(S_{i}, x\right) \rightarrow \pi_{1}(S) \cong H_{1}(S, \mathbb{Z}),
$$

where $x \in V \cap S_{i}$. Consequently $U_{n}$ must be nonempty, for otherwise we would obtain an open covering of the torus whose existence is ruled out by Corollary 2.2 This shows $\delta(x)=n$ for some $x \in S$, and thus $a \cdot L$ is well-rounded for some $a \in L$.

Remark. The stratification $\left(S_{i}\right)$ used above is actually dual to the stratification used in the proof sketched in the introduction.

\section{TOPOLOGY OF $\mathbb{R}^{n}$}

In this section we establish the following covering theorem for $\mathbb{R}^{n}$.

Theorem 5.1. Let $\mathfrak{U}$ be an open covering of $\mathbb{R}^{n}$ with inradius $r>0$, and let $R>0$. Suppose that every component $V$ of the intersection $U_{1} \cap U_{2} \cap \cdots \cap U_{k}$ of $1 \leq k \leq n$ distinct elements of $\mathfrak{U}$ is contained in an open set $F(V)$ isometric to $B^{k}(R) \times \mathbb{R}^{n-k}$. Then $\mathfrak{U}$ has order at least $n$.

Here $\mathfrak{U}$ has inradius $r$ if for every $p \in \mathbb{R}^{n}$, there exists a $U \in \mathfrak{U}$ with $B(p, r) \subset U$.

Note that Theorem 2.1 on coverings of $T^{n}$ follows from the result above, by passing to the universal cover. 
Bounded cohomology. The proof of Theorem [5.1 will use the cohomology of bounded differential forms to detect the 'fundamental class' of $\mathbb{R}^{n}$. We begin with a brief résumé of this theory.

Let $M$ be a Riemannian manifold, and let $|v|$ denote the length of a tangent vector $v \in \mathrm{T} M$. Then the $L^{\infty}$-norm of a smooth $k$-form on $M$ is given by

$$
\|\alpha\|_{\infty}=\sup _{\left|v_{1}\right|=\cdots=\left|v_{k}\right|=1}\left|\alpha\left(v_{1}, \ldots, v_{k}\right)\right| .
$$

We say a form is bounded if both $\alpha$ and $d \alpha$ are in $L^{\infty}$.

Let $\Omega_{b}^{*}(M)$ denote the space of smooth, bounded forms on $M$, equipped with the norm

$$
\|\alpha\|_{b}=\|\alpha\|_{\infty}+\|d \alpha\|_{\infty} .
$$

Since $d^{2}=0$, we have $\|d \alpha\|_{b} \leq\|\alpha\|_{b}$. Therefore $\left(\Omega_{b}^{*}(M), d\right)$ is a differential complex. The bounded cohomology groups of $M$ are given by

$$
H_{b}^{k}(M)=\frac{\left\{\alpha \in \Omega_{b}^{k}(M): d \alpha=0\right\}}{d \Omega_{b}^{k-1}(M)} .
$$

See [Gr for the analogous case of bounded singular cohomology and Pan for an exposition of $L^{2}$-cohomology.

Invariance properties. Any smooth map $f: M \rightarrow N$ between Riemannian manifolds that satisfies

$$
\|D f\|_{\infty}=\sup _{v \neq 0}|D f(v)| /|v|<\infty
$$

induces a natural map $f^{*}: H_{b}^{*}(N) \rightarrow H_{b}^{*}(M)$. In particular, if $f$ is a quasi-isometric diffeomorphism, $f^{*}$ is an isomorphism on bounded cohomology.

The Poincaré lemma. The cohomology groups $H_{b}^{*}(M)$ are insensitive to thickenings of $M$. To make this precise, let $I=(-1,1)$ and let $M \times I$ be given the product metric. Let $s: M \rightarrow M \times\{0\}$ be the natural section of the projection map $p: M \times I \rightarrow M$. Then we have

Lemma 5.2. The groups $H_{b}^{*}(M \times I)$ and $H_{b}^{*}(M)$ are isomorphic. In fact, for each $k$ there is an operator

$$
K: \Omega_{b}^{k}(M \times I) \rightarrow \Omega_{b}^{k-1}(M \times I),
$$

satisfying $\|K\| \leq 2$, with $\mathrm{Id}-p^{*} \circ s^{*}=(-1)^{k}(d K-K d)$.

Proof. We first recall the proof for deRham cohomology [BT, §4]. Any $k$-form on $M \times I$ can be written as

$$
\omega=g(x, t) \alpha+f(x, t) d t \wedge \beta,
$$

where $\alpha=p^{*}\left(\alpha^{\prime}\right)$ and $\beta=p^{*}\left(\beta^{\prime}\right)$ are the pullbacks of forms of degree $k$ and $k-1$ on $M$. Using this decomposition of $\omega$, we define

$$
K: \Omega_{b}^{k}(M \times I) \rightarrow \Omega_{b}^{k-1}(M \times I)
$$

by

$$
K(\omega)=\left(\int_{0}^{t} f(x, s) d s\right) \beta .
$$

Then the operator $K$ satisfies

$$
\omega-p^{*} \circ s^{*}(\omega)=(-1)^{k}(d K \omega-K d \omega),
$$


which implies $p^{*} \circ s^{*}$ gives an isomorphism on the level of deRham cohomology. The opposite composition satisfies $s^{*} \circ p^{*}=\mathrm{Id}$, and therefore the deRham cohomology groups of $M$ and $M \times I$ are isomorphic.

Now observe that if $\omega$ is bounded, then so is $K \omega$. In fact, we have

$$
\|K \omega\|_{\infty} \leq\|\omega\|_{\infty}
$$

because $\left|\int_{0}^{t} f(s) d s\right| \leq \sup |f|$; and by (5.1), we have

$$
\|d K \omega\|_{\infty} \leq\|\omega\|_{\infty}+\left\|p^{*} s^{*} \omega\right\|_{\infty}+\|K d \omega\|_{\infty} \leq 2\|\omega\|_{\infty}+\|d \omega\|_{\infty},
$$

which implies $\|K \omega\|_{b} \leq 2\|\omega\|_{b}$. Therefore $K$ also defines a chain homotopy on the complex of bounded forms, showing that the bounded cohomology groups $H_{b}^{*}(M)$ and $H_{b}^{*}(M \times I)$ are isomorphic as well.

Examples. The bounded cohomology of $\mathbb{R}^{n}$ is nontrivial in every dimension $0 \leq$ $k \leq n$. For example, the cohomology class $[\omega]=\left[d x_{1} \cdots d x_{n}\right]$ is nonzero because $\int_{B^{n}(R)} \omega=C_{n} R^{n}$ as $R \rightarrow \infty$, while

$$
\int_{B^{n}(R)} d \eta=\int_{S^{n-1}(R)} \eta=O\left(R^{n-1}\right)
$$

for any bounded $(n-1)$-form $\eta$. The same reasoning shows $\left[d x_{1} \cdots d x_{k}\right] \neq 0$.

On the other hand, the bounded cohomology of a ball, $H_{b}^{k}\left(B^{n}\right)$, vanishes for all $k>0$. This follows from the fact that $B^{n}$ is quasi-isometric to $I^{n}$, and $H_{b}^{*}\left(I^{n}\right) \cong$ $H_{b}^{*}\left(\mathbb{R}^{0}\right)$. Similarly, for any $n$-dimensional manifold we have

$$
H_{b}^{n+i}\left(B^{j} \times M^{n}\right)=0
$$

for all $i, j>0$.

A uniform vanishing theorem. By iterating Lemma 5.2 , we obtain the following quantitative version of (5.2):

Theorem 5.3. Let $\omega$ be a closed, bounded $(n+i)$-form on $N=B^{j} \times M^{n}, i, j>0$. Then there is a solution to the equation $d \eta=\omega$ on $N$ satisfying

$$
\|\eta\|_{b} \leq C(n+j)\|\omega\|_{b}
$$

The constant $C(n+j)$ is independent of $M^{n}$.

Cohomology via covers of $\mathbb{R}^{n}$. To adapt the Čech-deRham complex to bounded forms, we must add a uniformity condition to the definition of cochains. Namely, we define

$$
C^{p}\left(\mathfrak{U}, \Omega_{b}^{q}\right)=\bigoplus_{|I|=p+1} \Omega_{b}^{p}\left(U_{I}\right)
$$

to be a direct sum in the category of normed spaces; its elements are those chains such that

$$
\left\|\left(\omega_{I}\right)\right\|=\sup _{I}\left\|\omega_{I}\right\|_{b}
$$

is finite. Using this norm, the differentials $d$ and $\delta$ become bounded operators on the double complex $C^{*}\left(\mathfrak{U}, \Omega_{b}^{*}\right)$. We also obtain a diagonal complex

$$
A^{r}=\bigoplus_{p+q=r} C^{p}\left(\mathfrak{U}, \Omega_{b}^{q}\right)
$$

as before, with differential $D=\delta+(-1)^{p} d$. 
Theorem 5.4. Let $\mathfrak{U}$ be an open covering of $\mathbb{R}^{n}$ with inradius $r>0$ and finite order. Then $H_{b}^{*}\left(\mathbb{R}^{n}\right)$ is isomorphic to $H_{D}^{*}\left(C^{*}\left(\mathfrak{U}, \Omega_{b}^{*}\right)\right)$.

Proof. Because of the inradius and order conditions, we can find a partition of unity $\sum \rho_{i}=1$ subordinate to the cover $U_{i}$, with $0 \leq \rho_{i} \leq 1$ and $\sup _{i}\left\|d \rho_{i}\right\|_{\infty}<\infty$. The operators $\alpha \mapsto \rho_{i} \alpha$ on $\Omega_{b}^{p}$ are then uniformly bounded. Thus one can apply the usual proof of the vanishing of the cohomology of fine sheaves [BT, Prop. 8.5] to show the sequence

$$
C^{0}\left(\mathfrak{U}, \Omega_{b}^{q}\right) \stackrel{\delta}{\rightarrow} C^{1}\left(\mathfrak{U}, \Omega_{b}^{q}\right) \stackrel{\delta}{\rightarrow} C^{2}\left(\mathfrak{U}, \Omega_{b}^{q}\right) \stackrel{\delta}{\rightarrow} \cdots
$$

is exact for every $q$. Consequently every cohomology class in $H_{D}^{k}\left(C^{*}\left(\mathfrak{U}, \Omega_{b}^{*}\right)\right)$ is represented by a cochain in $C^{0}\left(\mathfrak{U}, \Omega_{b}^{k}\right)$, and the theorem follows.

See [Pan, Ch. 4] for a similar result in the $L^{2}$ case.

Envelopes. Now let $\mathfrak{U}=\left\{U_{i}\right\}$ be an open covering of $\mathbb{R}^{n}$ satisfying the conditions of Theorem [5.1. Let $I$ range over index sets with $|I|=k, 1 \leq k \leq n$, and let $V$ range over the components of $U_{I}$. By hypothesis, $V$ is contained in an open set $F(V) \subset \mathbb{R}^{n}$ isometric to $B^{k}(R) \times \mathbb{R}^{n-k}$.

Let $R_{k}=10^{n-k+1} R$. We will construct open sets $E(V) \subset \mathbb{R}^{n}$ and $M^{n-k}(V) \subset$ $\mathbb{R}^{n-k}$ such that

$\left(E_{1}\right) V \subset E(V)$;

$\left(E_{2}\right) E(V)$ is isometric to $B^{k}\left(R_{k}\right) \times M^{n-k}(V)$; and

$\left(E_{3}\right) V^{\prime} \subset V \Longrightarrow E\left(V^{\prime}\right) \subset E(V)$, whenever $V^{\prime}$ is a component of $U_{J}$ and $|J|=k+1$.

We refer to $E(V)$ as the envelope of $V$.

To construct $E(V)$, consider the unique product structure

$$
\mathbb{R}^{n} \cong \mathbb{R}^{k} \times \mathbb{R}^{n-k}
$$

extending the isometry $F(V) \cong B^{k}(R) \times \mathbb{R}^{n-k}$. In these coordinates, we define

$$
E(V)=B^{k}\left(R_{k}\right) \times M^{n-k}(V),
$$

where $M^{n-k}(V)$ is an $R_{k}$-neighborhood of the projection of $V$ to the $\mathbb{R}^{n-k}$ factor in (5.4).

By construction, the sets $V$ and $E(V)$ are approximately equal at scale $R_{k}$. More precisely, we have

$$
B\left(V, R_{k} / 2\right) \subset E(V) \subset B\left(V, 3 R_{k}\right) .
$$

Conditions $\left(E_{1}\right)$ and $\left(E_{2}\right)$ above are now immediate. To verify $\left(E_{3}\right)$, simply note that if $V^{\prime}$ is a component of $U_{J}$, with $|J|=k+1$ and $V^{\prime} \subset V$, then (5.5) implies

$$
E\left(V^{\prime}\right) \subset B\left(V^{\prime}, 3 R_{k+1}\right) \subset B\left(V, R_{k} / 2\right) \subset E(V) .
$$

Enveloping forms. We define the normed space of enveloping forms for $V$ by

$$
\mathcal{F}_{b}^{q}(V)=\Omega_{b}^{q}(E(V)) .
$$

These are forms on $V$ equipped with a bounded extension to the envelope $E(V)$. We extend the definition to $U_{I}$ by setting

$$
\mathcal{F}_{b}^{q}\left(U_{I}\right)=\bigoplus \Omega_{b}^{q}(V),
$$


where the sum is over the components of $U_{I}$; and finally we define

$$
C^{p}\left(\mathfrak{U}, \mathcal{F}_{b}^{q}\right)=\bigoplus_{|I|=p+1} \mathcal{F}_{b}^{q}\left(U_{I}\right)
$$

As in (5.3), both direct sums above are taken in the category of normed spaces.

The operators $d$ and $\delta$ make $C^{*}\left(\mathfrak{U}, \mathcal{F}_{b}^{*}\right)$ into a chain complex, and the restriction maps $\mathcal{F}_{b}^{*}\left(U_{I}\right) \rightarrow \Omega^{*}\left(U_{I}\right)$ determine a natural map

$$
H_{D}^{*}\left(C^{*}\left(\mathfrak{U}, \mathcal{F}_{b}^{*}\right)\right) \rightarrow H_{D}^{*}\left(C^{*}\left(\mathfrak{U}, \Omega_{b}^{*}\right)\right)
$$

on the level of cohomology.

Proof of Theorem 5.1. Let $\mathfrak{U}$ be a covering of $\mathbb{R}^{n}$ satisfying the hypotheses of the theorem, but with order less than $n$. We will deduce a contradiction, by showing the bounded cohomology class

$$
[\omega]=\left[d x_{1} \cdots d x_{n}\right] \in H_{b}^{n}\left(\mathbb{R}^{n}\right)
$$

is zero.

To begin with, note that $[\omega]$ lifts to a class $\left[y_{0}\right] \in H_{D}^{n}\left(C^{*}\left(\mathfrak{U}, \mathcal{F}_{b}^{*}\right)\right)$, represented by the cocycle

$$
y_{0}=(\omega \mid E(V)) \in C^{0}\left(\mathfrak{U}, \mathcal{F}^{n}\right) .
$$

This class $\left[y_{0}\right]$ projects back to $[\omega]$ under the composition of $(\underline{5.6}$ with the isomorphism

$$
H_{D}^{n}\left(C^{*}\left(\mathfrak{U}, \Omega_{b}^{*}\right)\right) \cong H_{b}^{n}\left(\mathbb{R}^{n}\right)
$$

provided by Theorem 5.4 Thus to complete the proof, it suffices to show $\left[y_{0}\right]=0$ in $H_{D}^{n}\left(C^{*}\left(\mathfrak{U}, \mathcal{F}_{b}^{*}\right)\right)$.

We first show the sequence

$$
C^{p}\left(\mathfrak{U}, \mathcal{F}_{b}^{q-1}\right) \stackrel{d}{\rightarrow} C^{p}\left(\mathfrak{U}, \mathcal{F}_{b}^{q}\right) \stackrel{d}{\rightarrow} C^{p}\left(\mathfrak{U}, \mathcal{F}_{b}^{q+1}\right)
$$

is exact, whenever $p+q=n$. To see this, suppose $\left(\omega_{V, I}\right)$ is an element of the central term above, with $\omega_{V, I} \in \Omega_{b}^{q}(E(V))$ and $d \omega_{V, I}=0$. Then for each $V$, the envelope $E(V)$ is isometric to $B^{k}\left(R_{k}\right) \times M^{q-1}(V)$, where $k=p+1$. Since $R_{k} \leq 10^{n+1} R$, by Theorem 5.3 we can solve the equation $\omega_{V, I}=d \eta_{V, I}$ on $E(V)$ with a uniform bound

$$
\left\|\eta_{V, I}\right\|_{b} \leq C(R)\left\|\omega_{V, I}\right\|_{b}
$$

Thus $\left(\eta_{V, I}\right)$ defines a chain in $C^{p}\left(\mathfrak{U}, \mathcal{F}_{b}^{q-1}\right)$ mapping under $d$ to $\left(\omega_{V, I}\right)$, and exactness is established.

It follows, just as in the proof of Theorem 2.1, that the cocycle $y_{0}$ is cohomologous to a cycle $y_{n} \in C^{n}\left(\mathfrak{U}, \mathcal{F}_{b}^{0}\right)$. But since $\mathfrak{U}$ has order less than $n$, this chain group vanishes, and therefore $\left[y_{0}\right]=\left[y_{n}\right]=0$ in $H_{D}^{n}\left(C^{*}\left(\mathfrak{U}, \mathcal{F}_{b}^{*}\right)\right)$.

\section{INCOMPRESSIBLE SUBSPACES}

In this section we introduce the theory of incompressible subspaces $M \subset \mathbb{R}^{n}$, in preparation for studying general lattices with $N(L)>0$. 
Norm and support. Let $e_{i}, i \in I=\{1, \ldots, n\}$, be the standard basis for $\mathbb{R}^{n}$. Then the elements $e_{J}=e_{j_{1}} \wedge e_{j_{2}} \wedge \cdots \wedge e_{j_{k}}$ give a basis for $\wedge^{k}\left(\mathbb{R}^{n}\right)$, as $J$ ranges over all increasing sequences $\left(j_{1}, \ldots, j_{k}\right) \subset I$. We define the norm and support of an element

$$
\omega=\sum_{|J|=k} m_{J} e_{J} \in \wedge^{k}\left(\mathbb{R}^{n}\right)
$$

by $\|\omega\|=\sup _{|J|=k}\left|m_{J}\right|$ and $\operatorname{supp}(\omega)=\left\{J: m_{J} \neq 0\right\}$.

Measured subspaces and discrete groups. A $k$-dimensional measured subspace is a real linear subspace $M \subset \mathbb{R}^{n}$ equipped with a nonzero volume element

$$
\operatorname{det}(M) \in \wedge^{k}(M)
$$

well-defined up to sign.

The set of all such $M$ is naturally parameterized by the space

$$
\mathcal{G}_{n, k}=\mathrm{GL}_{n, k}(\mathbb{R}) / \mathrm{SL}_{k}^{ \pm}(\mathbb{R}),
$$

where $\mathrm{SL}_{k}^{ \pm}(\mathbb{R})$ is the group of $k \times k$ matrices with determinant \pm 1 , and $\mathrm{GL}_{n, k}(\mathbb{R})$ is the space of linear maps $g: \mathbb{R}^{k} \rightarrow \mathbb{R}^{n}$ of maximal rank. The measured subspace corresponding to $[g] \in \mathcal{G}_{n, k}$ is simply $M=g\left(\mathbb{R}^{k}\right)$, with $\operatorname{det}(M)=g\left(e_{1}\right) \wedge \cdots \wedge g\left(e_{k}\right)$.

Note that $\mathcal{G}_{n, k}$ is an $\mathbb{R}_{+}$-bundle over the compact Grassmann variety of $k$-planes in $\mathbb{R}^{n}$. In particular,

$$
\bar{E} \subset \mathcal{G}_{n, k} \text { is compact } \Longleftrightarrow 0<\inf _{E}\|\operatorname{det}(M)\| \leq \sup _{E}\|\operatorname{det}(M)\|<\infty .
$$

Compressibility. A measured subspace $M \subset \mathbb{R}^{n}$ is compressible if there is a sequence $a_{n} \in A$ such that $\left\|\operatorname{det}\left(a_{n} \cdot M\right)\right\| \rightarrow 0$. Otherwise $M$ is incompressible. Note that compressibility depends only on the support of $M$, and it makes sense to refer to an incompressible subspace.

The principal result of this section shows that if $M$ is incompressible, then $\operatorname{det}(a \cdot M)$ is large outside a set of the form $B^{i} \times \mathbb{R}^{j} \subset A$, with $j \leq \operatorname{gcd}(k, n)-1$.

Theorem 6.1. Given $0<k \leq n$, there exist

- connected subgroups $H_{1}, \ldots, H_{N}$ of $A$, with $\operatorname{dim} H_{i} \leq \operatorname{gcd}(k, n)-1$, and

- compact sets $B_{R} \subset A$, indexed by $R \geq 1$,

such that for any incompressible $M \in \mathcal{G}_{n, k}$ with $\inf _{A}\|\operatorname{det}(a \cdot M)\|=1$, we have

$$
\{a \in A:\|\operatorname{det}(a \cdot M)\| \leq R\} \subset a_{0} \cdot B_{R} \cdot H_{i}
$$

for some subgroup $H_{i}$ and some $a_{0} \in A$.

Rank of the stabilizer We denote the stabilizer of $M \in \mathcal{G}_{k, n}$ by

$$
A_{M}=\{a \in A: a \cdot M=M \text { and } \operatorname{det}(a \mid M)=1\} ;
$$

it is a connected subgroup isomorphic to $\mathbb{R}_{+}^{d}$ for some $d$. The first step in the proof of Theorem 6.1 is to control the size of $A_{M}$. Note that the bound we obtain parallels Theorem 4.7 from the number field case.

Theorem 6.2. If $M \subset \mathbb{R}^{n}$ is an incompressible subspace of dimension $k>0$, then we have $\operatorname{dim} A_{M} \leq \operatorname{gcd}(k, n)-1$. 
Proof. We claim that $A_{M}=\{a \in A: a \cdot M=M\}$. Indeed, if $a \cdot M=M$ and $\operatorname{det}(a \mid M) \neq 1$, then either $\left\|\operatorname{det}\left(a^{n} \cdot M\right)\right\| \rightarrow 0$ or $\left\|\operatorname{det}\left(a^{-n} \cdot M\right)\right\| \rightarrow 0$ as $n \rightarrow+\infty$, contradicting incompressibility of $M$.

Now suppose $A_{M}$ has dimension $s-1$. Then the eigenspaces of a regular element of $g \in A_{M}$ determine a splitting $\mathbb{R}^{n}=\bigoplus_{1}^{s} V_{i}$, with $n_{i}=\operatorname{dim} V_{i}>0$ and $\sum n_{i}=n$. Since $g \cdot M=M$, we can also write $M$ as a direct sum of eigenspaces $M_{i}=M \cap V_{i}$, and therefore

$$
A_{M}=\left\{a=\bigoplus_{1}^{s} a_{i} \cdot \operatorname{Id}_{V_{i}}: \prod a_{i}^{n_{i}}=1\right\} .
$$

Note that $k_{i}=\operatorname{dim} M_{i}$ satisfies $\sum k_{i}=k$. Since

$$
\operatorname{det}(a \mid M)=\prod a_{i}^{k_{i}}=1
$$

for all $a \in A_{M}$, the exponents $\left(n_{i}\right)$ and $\left(k_{i}\right)$ must be integral multiples of a common primitive integral vector $\left(p_{i}\right)$. Consequently $p=\sum_{1}^{s} p_{i}$ divides both $n=\sum n_{i}$ and $k=\sum k_{i}$, and therefore $s=\operatorname{dim} A_{M}+1=\sum_{1}^{s} 1 \leq p \leq \operatorname{gcd}(k, n)$.

Strict incompressibility. We say $M$ is strictly incompressible if the function

$$
\|\operatorname{det}(a \cdot M)\|: A / A_{M} \rightarrow \mathbb{R}
$$

is proper.

By convex duality, $M$ is incompressible iff there exist $\alpha_{J} \geq 0$ such that $\chi_{I}=$ $\sum_{J \in \operatorname{supp}(M)} \alpha_{J} \chi_{J}$, and it is strictly incompressible if we can take $\alpha_{J}>0$. (Here $\chi_{J}: I \rightarrow\{0,1\}$ is the indicator function of $J \subset I$.)

Examples. Consider the measured subspaces of $\mathbb{R}^{4}$ given by

$$
\begin{aligned}
& \operatorname{det}\left(M_{1}\right)=\left(e_{1}+e_{2}+e_{3}\right) \wedge e_{4}, \\
& \operatorname{det}\left(M_{2}\right)=\left(e_{1}+e_{2}+e_{3}\right) \wedge\left(e_{3}+e_{4}\right), \quad \text { and } \\
& \operatorname{det}\left(M_{3}\right)=\left(e_{1}+e_{2}\right) \wedge\left(e_{3}+e_{4}\right) .
\end{aligned}
$$

Since $\operatorname{det}\left(a^{n} M_{1}\right) \rightarrow 0$ for $a=\operatorname{diag}(2,2,2,1 / 8), M_{1}$ is compressible. The measured subspace $M_{2}$ is incompressible, but not strictly so: for $a=\operatorname{diag}(2,2,1 / 2,1 / 2)$, $\left\|\operatorname{det}\left(a^{n} \cdot M_{2}\right)\right\|$ stays bounded as $n \rightarrow \infty$, but $a \cdot M_{2} \neq M_{2}$. The subspace $M_{3}$ is strictly incompressible.

Theorem 6.3. If $M$ is incompressible, then there exists a strictly incompressible measured subspace $M^{\prime}$ with supp $M^{\prime} \subset \operatorname{supp} M$.

Proof. We can take $M=M^{\prime}$ if $M$ itself is strictly incompressible.

Otherwise, there is an $a \in A$ such that $a_{J} \leq 1$ for all $J \in \operatorname{supp}(M)$ and strict inequality holds for some multi-index $J^{\prime}$. This implies $\left\|\operatorname{det}\left(a^{n} M\right)\right\|$ is bounded above by $\|\operatorname{det}(M)\|$; it is also bounded below (away from zero), by incompressibility of $M$. Thus the compactness criterion (6.1) implies $a^{n} \cdot M$ accumulates on some $M^{\prime} \in \mathcal{G}_{n, k}$.

Since $A \cdot M^{\prime} \subset \overline{A \cdot M}, M^{\prime}$ is also incompressible; and by construction, the support of $M^{\prime}$ is strictly smaller than that of $M$ (it excludes $J^{\prime}$ ) . But the support cannot shrink indefinitely, so after iterating this process a finite number of steps, we obtain a strictly incompressible subspace with $\operatorname{supp}\left(M^{\prime}\right) \subset \operatorname{supp}(M)$. 
Proof of Theorem 6.1. There are only a finite number of possibilities for the support of a $k$-dimensional subspace of $\mathbb{R}^{n}$. Thus we can choose $M_{1}, \ldots, M_{N} \in \mathcal{G}_{n, k}$, such that every strictly incompressible $M \in \mathcal{G}_{n, k}$ satisfies $\operatorname{supp} M=\operatorname{supp} M_{i}$ for some $i$. Let $H_{i}=A_{M_{i}}$.

Next, consider any $M_{0}$ in the compact set of incompressible subspaces

$$
\mathcal{M}_{n, k}=\left\{M \in \mathcal{G}_{n, k}:\|\operatorname{det}(M)\| \leq 2 \text { and } \inf _{A}\|\operatorname{det}(a \cdot M)\|=1\right\} .
$$

By the preceding theorem, we have $\operatorname{supp}\left(M_{i}\right) \subset \operatorname{supp}\left(M_{0}\right)$ for some $i$. Let $U \subset \mathcal{G}_{n, k}$ be the open set defined by

$$
U=\left\{M: \operatorname{det}(M)=\sum m_{J} e_{J} \text { with } \min _{J \in \operatorname{supp} M_{i}}\left|m_{J}\right|>\epsilon\right\},
$$

where $\epsilon>0$ is chosen so that $M_{0} \in U$. There is a constant $C>0$ such that

$$
\|\operatorname{det}(a \cdot M)\| \geq C \cdot\left\|\operatorname{det}\left(a \cdot M_{i}\right)\right\|
$$

for all $M \in U$ and $a \in A$.

Since $M_{i}$ is strictly incompressible, the map $\left\|\operatorname{det}\left(a \cdot M_{i}\right)\right\|: A / H_{i} \rightarrow \mathbb{R}$ is proper. Thus there is a compact set $B_{R, U} \subset A$ such that

$$
\left\{a \in A:\left\|\operatorname{det}\left(a \cdot M_{i}\right)\right\| \leq C^{-1} \cdot R\right\} \subset B_{R, U} \cdot H_{i} .
$$

By (6.3), we then have

$$
\{a \in A:\|\operatorname{det}(a \cdot M)\| \leq R\} \subset B_{R, U} \cdot H_{i}
$$

for all $M \in U$. Since $\mathcal{M}_{n, k}$ is compact, we can take the union of the sets $B_{R, U}$ over a finite cover to obtain a compact set $B_{R}$ that works simultaneously for all $M_{0} \in \mathcal{M}_{n, k}$. It follows that (6.2) holds for any measured subspace with $\inf _{A}\|\operatorname{det}(a \cdot M)\|=1$, by choosing $a_{0}$ such that $a_{0}^{-1} M \in \mathcal{M}_{n, k}$.

Remark. Only certain collections of multi-indices $J$ can arise as $J=\operatorname{supp}(M)$ for some $M \subset \mathbb{R}^{n}$, but the set of $J$ that do arise is very difficult to describe explicitly Va].

\section{PROOF FOR BOUNDED ORBITS}

In this section we complete the proof of our main result, by showing

Theorem 7.1. If $L \subset \mathbb{R}^{n}$ is a unimodular lattice with $N(L)>0$, then $\overline{A \cdot L}$ contains a well-rounded lattice.

The idea of the proof is to define a covering $U_{1}, \ldots, U_{n}$ of $A \cong \mathbb{R}^{n-1}$ determined by the span of the minimal vectors of $a \cdot L$ (as in the case of a compact orbit), and then to apply Theorem 5.1 to deduce $U_{n} \neq \emptyset$.

Notation. We will write $A=O(B)$ and $A \asymp B$ to indicate that $A \leq C_{n} B$ and $A / C_{n} \leq B \leq C_{n} A$, where $C_{n}$ depends only on the dimension $n$. 
Metrics. We begin by introducing a translation-invariant metric $d(a, b)$ on $A$.

Let $\operatorname{Lie}(A)$ be the Lie algebra of $A$, regarded as the space of vectors $\left(t_{1}, \ldots, t_{n}\right) \in$ $\mathbb{R}^{n}$ such that $\sum t_{i}=0$. Let exp : $\operatorname{Lie}(A) \rightarrow A$ be the exponential map, defined by

$$
\exp (t)=\operatorname{diag}\left(e^{t_{1}}, \ldots, e^{t_{n}}\right),
$$

let $\log : A \rightarrow \operatorname{Lie}(A)$ be its inverse, and finally define

$$
d(a, b)=|\log (a)-\log (b)| .
$$

Clearly $(A, d)$ is isometric to the Euclidean space $\mathbb{R}^{n-1}$. We also have $d(a, b) \asymp$ $\log \left\|a^{-1} b\right\|$, where $\left\|\operatorname{diag}\left(a_{1}, \ldots, a_{n}\right)\right\|=\sup \left|a_{i}\right|$ is the operator norm on $A$.

Almost minimal vectors. Given a $L \subset \mathbb{R}^{n}$ and $r \geq 0$, let $\operatorname{span}_{r}(L) \subset \mathbb{R}^{n}$ denote the real vector space spanned by

$$
\{y \in L:|y| \leq(1+r)|L|\} .
$$

Note that $L$ is well-rounded iff $\operatorname{span}_{0}(L)=\mathbb{R}^{n}$, and when $r>0$ is small, the generators of $\operatorname{span}_{r}(L)$ are almost minimal vectors for $L$.

Let $\operatorname{dim}_{r}(L)=\operatorname{dim} \operatorname{span}_{r}(L)$. Then $\operatorname{dim}_{r}(L)$ is an increasing function of $r$, with at most $(n-1)$ jump discontinuities. Away from these jumps, $\operatorname{dim}_{r}(L)$ is stable as $L$ varies; more precisely, if $0<r<s<t$ are given, then there is a neighborhood $U$ of the identity in $A$ such that

$$
\operatorname{dim}_{r}(L) \leq \operatorname{dim}_{s}(a \cdot L) \leq \operatorname{dim}_{t}(L)
$$

for all lattices $L$ and all $a \in U$.

Covering $A$. We now define a covering $\left(U_{k}\right)_{1}^{n}$ of $A$, depending on a parameter $0<\epsilon<1$, by

$$
U_{k}=\left\{a \in A: \operatorname{dim}_{r}(a \cdot L)=k \text { for all } r \text { in a neighborhood of } r=k \epsilon\right\} .
$$

Theorem 7.2. The sets $U_{1}, \ldots, U_{n}$ give an open covering of $A \cong \mathbb{R}^{n-1}$ with inradius bounded below.

Proof. The sets $\left(U_{k}\right)$ are open by stability of the function $\operatorname{dim}_{r}(a \cdot L)$. To see they cover, given $a \in A$ consider the least $k \geq 1$ such that $\operatorname{dim}_{(k+1 / 2) \epsilon}(a \cdot L) \leq k$. Then $k \leq n$ and $\operatorname{dim}_{(k-1 / 2) \epsilon}(a \cdot L) \geq k$, so we have

$$
\operatorname{dim}_{r}(L)=k \text { for }(k-1 / 2) \epsilon \leq r \leq(k+1 / 2) \epsilon .
$$

Thus $a$ belongs to $U_{k}$. Moreover, by (7.1), there is a neighborhood $U$ of the identity in $A$, depending only on $\epsilon$, such that $a \cdot U \subset U_{k}$ as well. Thus the inradius of the covering is bounded below.

Incompressibility. We now bring the ideas of the preceding section into play.

Let $P=\mathbb{Z} y_{1} \oplus \cdots \oplus \mathbb{Z} y_{k}$ be a discrete subgroup of $\mathbb{R}^{n}$, of rank $k>0$. Let $|P|$ and $N(P)$ denote the minimum of $|y|$ and $N(y)$ over nonzero vectors $y \in P$. The determinant of $P$ is defined by

$$
\operatorname{det}(P)=y_{1} \wedge \cdots \wedge y_{k} \in \wedge^{k}\left(\mathbb{R}^{n}\right) ;
$$

it is well-defined up to sign, and satisfies

$$
\|\operatorname{det}(P)\| \asymp \operatorname{vol}(P \otimes \mathbb{R} / P) .
$$


Since the quotient torus $(P \otimes \mathbb{R}) / P$ contains an embedded ball of diameter $|P|$, we also have Minkowski's bound

$$
|P|^{k}=O(\|\operatorname{det}(P)\|) .
$$

Let $M_{k}: U_{k} \rightarrow \mathcal{G}_{n, k}$ be the function sending $a \in U_{k}$ to the $k$-dimensional subspace

$$
M_{k}(a)=a^{-1} \cdot \operatorname{span}_{k \epsilon}(a \cdot L),
$$

with its determinant defined by $\operatorname{det} M_{k}(a)=\operatorname{det}\left(M_{k}(a) \cap L\right)$. The vectors in $L$ spanning $M_{k}(a)$ are locally constant, so $M_{k}(a)$ is itself a locally constant function on $U_{k}$.

Theorem 7.3. The measured subspace $M_{k}(a)$ is incompressible. More precisely, we have $\left\|\operatorname{det}\left(a \cdot M_{k}(a)\right)\right\|=O(1)$ and

$$
\left\|\operatorname{det}\left(b \cdot M_{k}(a)\right)\right\| \geq c(N(L))>0
$$

for all $a \in U_{k}$ and $b \in A$

(Here $c(N(L))$ is a constant depending only on $N(L)$.)

Proof. Given $a \in U_{k}$, let $M=M_{k}(a)$ and let $P=M \cap L$. Then by the definition of $\operatorname{span}_{k \epsilon}(a \cdot L)$, there are linearly independent lattice vectors $y_{1}, \ldots, y_{k} \in P$ such that $\left|a y_{i}\right|=O(|a \cdot L|)$. Since $L$ is unimodular, we have $|\operatorname{det}(a \cdot L)|=1$ and thus $|a \cdot L|=O(1)$ by (7.3); therefore

$$
\|\operatorname{det}(a \cdot M)\| \leq\left\|\left(a y_{1}\right) \wedge \cdots \wedge\left(a y_{m}\right)\right\|=O(1) .
$$

For the lower bound, note that $N(b \cdot P)=N(P) \geq N(L)>0$ for any $b \in A$, and thus

$$
\sqrt{n} \cdot N(L)^{1 / n} \leq|b \cdot P|
$$

by the inequality between the arithmetic and geometric means. Applying (17.3) again, we find that $\|\operatorname{det}(b \cdot P)\|=\|\operatorname{det}(b \cdot M)\|$ is bounded below by a constant $c(N(L))$ depending only on $N(L)$.

Theorem 7.4. There is a constant $R>0$ such that every component $V$ of $U_{k}$ lies in a region $F(V) \subset A$ isometric to $B^{i}(R) \times \mathbb{R}^{j}$, where $j \leq \operatorname{gcd}(k, n)-1$ and $i+j=n-1$.

Proof. Let $V$ be a component of $U_{k}$. Since $M_{k}(a)$ is continuous, it assumes a constant value $M$ on $V$. By the preceding result, we can rescale $\operatorname{det}(M)$ so that $\inf _{A}\|\operatorname{det}(a \cdot M)\| \geq 1$ and for all $a \in V$ we have

$$
\left\|\operatorname{det}\left(a \cdot M_{k}(a)\right)\right\|=\|\operatorname{det}(a \cdot M)\| \leq S,
$$

where $S$ depends only on $N(L)$. Then by Theorem 6.1, there is a compact set $K_{S} \subset A$ such that

$$
V \subset\{a:\|\operatorname{det}(a \cdot M)\| \leq S\} \subset a_{0} \cdot K_{S} \cdot H
$$

for some $a_{0} \in A$ and some $j$-dimensional subgroup $H \subset A$ with $j \leq \operatorname{gcd}(k, n)-1$. Choose $R>0$ such that $K_{S}$ is contained in the ball $B(e, R)$ of radius $R$ about the identity $e \in A$. Then $V$ is contained in the region

$$
F(V)=a_{0} \cdot B(e, R) \cdot H .
$$

Since $\log (H) \subset \operatorname{Lie}(A)$ is isometric to $\mathbb{R}^{j}$, the region $F(V)$ is isometric to the product $B^{i}(R) \times \mathbb{R}^{j}$, where $i+j=\operatorname{dim} A=n-1$. 
Theorem 7.5. The open set $U_{n} \subset A$ is nonempty.

Proof. Suppose to the contrary that $U_{n}$ is empty. Let $\mathcal{U}$ be the open covering of $A \cong \mathbb{R}^{n-1}$ by the components of the sets $U_{1}, \ldots, U_{n-1}$. Then the inradius of $\mathcal{U}$ is bounded below. Moreover, if $V^{\prime}$ is a component of the intersection of $k$ distinct elements of $\mathcal{U}$, then $V^{\prime}$ is contained in a component $V$ of $U_{j}$ for some $j \leq n-k$. By the preceding result, we have

$$
V \subset F(V) \cong B^{k^{\prime}}(R) \times \mathbb{R}^{n-1-k^{\prime}}
$$

where $n-1-k^{\prime} \leq \operatorname{gcd}(j, n)-1 \leq n-k-1$. Thus $k^{\prime} \geq k$, and therefore $\mathcal{U}$ satisfies the hypotheses of the covering Theorem 5.1. Consequently $\mathcal{U}$ has order at least $n-1$ : it contains $n$ distinct elements whose intersection is nonempty. But any $n$ elements of $\mathcal{U}$ must include two distinct components from the same $U_{i}$, whose intersection is empty. This contradiction shows $U_{n} \neq \emptyset$.

Corollary 7.6. For any $\epsilon>0$, there exists an $L^{\prime} \in A \cdot L$ such that $\operatorname{dim}_{n \epsilon}\left(L^{\prime}\right)=n$.

Proof of Theorem 7.1. By the corollary above, for any $m>0$ there is a lattice $L_{m} \in A \cdot L$ such that $\operatorname{dim}_{1 / m}\left(L_{m}\right)=n$. Since $N(L)>0$, the sequence $L_{m}$ has an accumulation point $L^{\prime}$ in the compact set $\overline{A \cdot L}$. Then $\operatorname{span}_{1 / m}\left(L^{\prime}\right)=n$ for every $m>0$, so $\operatorname{span}_{0}\left(L^{\prime}\right)=n$ and $L^{\prime}$ is a well-rounded lattice.

\section{MinkOWsKI's CONJECTURE}

We conclude by showing that the main result implies

Theorem 8.1. Suppose that for all $k \leq n$, the covering radius of any well-rounded unimodular lattice $L \subset \mathbb{R}^{k}$ satisfies

$$
\operatorname{cov}(L) \leq \sqrt{k} / 2
$$

with equality only when $L \in \mathrm{SO}_{k}(\mathbb{R}) \cdot \mathbb{Z}^{k}$.

Then Minkowski's conjecture holds for all lattices in $\mathbb{R}^{k}, k \leq n$.

Covering radii. We denote the covering radii of a unimodular lattice $L$ with respect to $|x|$ and $N(x)$ by

$$
\operatorname{cov}(L)=\sup _{x \in \mathbb{R}^{n}} \inf _{y \in L}|x-y| \quad \text { and } \quad \operatorname{Ncov}(L)=\sup _{x \in \mathbb{R}^{n}} \inf _{y \in L} N(x-y) .
$$

Note that $\operatorname{cov}(L)$ is a continuous function of $L$, while $\operatorname{Ncov}(L)$ only satisfies the semicontinuity property

$$
\lim \sup \mathrm{N} \operatorname{cov}\left(L_{i}\right) \leq \mathrm{N} \operatorname{cov}\left(\lim L_{i}\right) .
$$

As usual, we have $\operatorname{Ncov}(L)^{1 / n} \leq \operatorname{cov}(L) / \sqrt{n}$.

Recall that Minkowski's conjecture $\left(M_{n}\right)$ asserts that all unimodular lattices $L \subset \mathbb{R}^{n}$ satisfy

$$
\operatorname{Ncov}(L) \leq \operatorname{Ncov}\left(\mathbb{Z}^{n}\right)=2^{-n},
$$

and equality holds iff $L \in A \cdot \mathbb{Z}^{n}$. Similarly, conjecture $\left(C_{n}\right)$ asserts that if $L$ is well-rounded, then

$$
\operatorname{cov}(L) \leq \operatorname{cov}\left(\mathbb{Z}^{n}\right)=\sqrt{n} / 2,
$$

and equality holds iff $L \in \mathrm{SO}_{n}(\mathbb{R}) \cdot \mathbb{Z}^{n}$.

For the proof of Theorem [8.1, we will use the following two results of Birch and Swinnerton-Dyer. 
Theorem 8.2. Minkowski's conjecture holds for all lattices in a neighborhood of $L=\mathbb{Z}^{n} \in \mathrm{SL}_{n}(\mathbb{R}) / \mathrm{SL}_{n}(\mathbb{Z})$.

Theorem 8.3. If Minkowski's conjecture holds for all lattices in $\mathbb{R}^{k}, k<n$, then it also holds for all lattices $L \subset \mathbb{R}^{n}$ with $N(L)=0$.

See [BiS, Theorems 1 and 2].

Proof of Theorem 8.1. Assuming $\left(C_{k}\right)$ holds for all $k \leq n$, we will show that Minkowski's conjecture $\left(M_{k}\right)$ also holds for all $k \leq n$. Proceeding inductively, we can assume $\left(M_{k}\right)$ holds for $k<n$, so we need only to establish $\left(M_{n}\right)$. By the result of Birch and Swinnerton-Dyer above, we need only to prove $\left(M_{n}\right)$ for lattices $L \subset \mathbb{R}^{n}$ with $N(L)>0$. But in this case, Theorem 7.1 shows there is a well-rounded lattice $L^{\prime} \in \overline{A \cdot L}$; by combining the inequalities (8.1) and (8.3), we then immediately obtain the bound

$$
\mathrm{Ncov}(L) \leq \mathrm{N} \operatorname{cov}\left(L^{\prime}\right) \leq\left(\operatorname{cov}\left(L^{\prime}\right) / \sqrt{n}\right)^{n} \leq 2^{-n}
$$

required for Minkowski's conjecture.

It remains to check the case of equality. First, observe that if $g \in \mathrm{SO}_{n}(\mathbb{R})$ and

$$
\operatorname{Ncov}\left(g \cdot \mathbb{Z}^{n}\right)^{1 / n}=\operatorname{cov}\left(\mathbb{Z}^{n}\right) / \sqrt{n},
$$

then $g \cdot \mathbb{Z}^{n}=\mathbb{Z}^{n}$. Indeed, the extreme values of $\operatorname{Ncov}\left(\mathbb{Z}^{n}\right)$ and $\operatorname{cov}\left(\mathbb{Z}^{n}\right)$ are both attained at the points $x=( \pm 1 / 2, \ldots, \pm 1 / 2)$, and if $g$ does not permute these points, then strict inequality holds in (8.5); cf. [Dy, I.3].

Now suppose equality holds in (8.2). Then the two inequalities in (8.4) are equalities, and consequently $L^{\prime}=g \cdot \mathbb{Z}^{n}=\mathbb{Z}^{n}$, since these are the cases of equality in (8.3) and (8.5).

Thus when $\operatorname{Ncov}(L)=2^{-n}$, the orbit $A \cdot L$ accumulates on the standard lattice $L^{\prime}=\mathbb{Z}^{n}$. Since Minkowski's conjecture holds in a neighborhood of $\mathbb{Z}^{n}$, we can only have equality when $L \in A \cdot \mathbb{Z}^{n}$, and the proof is complete.

\section{REFERENCES}

[Ash] A. Ash. Small-dimensional classifying spaces for arithmetical subgroups of general linear groups. Duke Math. J. 51(1984), 459-468. MF0747876 (85k:22027)

$[\mathrm{AM}]$ A. Ash and M. McConnell. Cohomology at infinity and the well-rounded retract for general linear groups. Duke Math. J. 90(1997), 549-576. MR1480546|(98h:11063)

[BW] R. P. Bambah and A. C. Woods. Minkowski's conjecture for $n=5$; a theorem of Skubenko. J. Number Theory 12(1980), 27-48. MR0566866 (81g:10043)

[Ba1] E. Bayer-Fluckiger. Lattices and number fields. In Algebraic Geometry: Hirzebruch 70 (Warsaw, 1998), pages 69-84. Amer. Math. Soc., 1999. MR1718137 (2000j:11049)

[Ba2] E. Bayer-Fluckiger. Ideal lattices. In A Panorama of Number Theory, pages 168-184. Cambridge Univ. Press, 2002. MR 1975451 (2004k:11108)

[BN] E. Bayer-Fluckiger and G. Nebe. On the Euclidean minimum of some real number fields. Preprint, 2004.

[BiS] B. J. Birch and H. P. F. Swinnerton-Dyer. On the inhomogeneous minimum of the product of $n$ linear forms. Mathematika 3(1956), 25-39. MR0079049 (18:22a)

[BoS] Z. I. Borevich and I. R. Shafarevich. Number Theory. Academic Press, 1966. MR0195803 (33:4001)

[BT] R. Bott and L. W. Tu. Differential Forms in Algebraic Topology. Springer-Verlag, 1982. MR0658304 (83i:57016)

[CaS] J. W. S. Cassels and H. P. F. Swinnerton-Dyer. On the product of three homogeneous linear forms and the indefinite ternary quadratic forms. Philos. Trans. Roy. Soc. London. Ser. A. 248(1955), 73-96. MR0070653(17:14f) 
[CoS] J. H. Conway and N. J. A. Sloane. Sphere Packings, Lattices and Groups. Springer-Verlag, 1999. MR1662447 (2000b:11077)

[Da] H. Davenport. A simple proof of Remak's theorem on the product of $n$ linear forms. $J$. London Math. Soc. 14(1939), 47-51.

[Dy] F. J. Dyson. On the product of four non-homogeneous linear forms. Annals of Math. 49(1948), 82-109. MR0025515(10:19a)

[EM] S. Eilenberg and N. Steenrod. Foundations of Algebraic Topology. Princeton University Press, 1952. MR0050886(14:398b)

[Gr] M. Gromov. Volume and bounded cohomology. IHES Publ. Math. 56(1982), 5-100. MR0686042 (84h:53053)

[GL] P. M. Gruber and C. G. Lekkerkerker. Geometry of Numbers. Elsevier, 1987. MR0893813 (88j:11034)

[HW] W. Hurewicz and H. Wallman. Dimension Theory. Princeton University Press, 1941. MR0006493 (3:312b)

[Ko] J. F. Koksma. Diophantische Approximationen. Springer-Verlag, 1936. MR0344200 (49:8940)

[LW] E. Lindenstrauss and B. Weiss. On sets invariant under the action of the diagonal group. Ergodic Theory Dynam. Systems 21(2001), 1481-1500. MF.1855843 (2002j:22009)

[Mg] G. A. Margulis. Problems and conjectures in rigidity theory. In Mathematics: Frontiers and Perspectives, pages 161-174. Amer. Math. Soc., 2000. MR1754775 (2001d:22008)

[Min] H. Minkowski. Diophantische Approximationen. Chelsea, 1957. MR0086102 (19:124f)

[Oh] H. Oh. Finiteness of compact maximal flats of bounded volume. Ergodic Theory Dynam. Systems 24(2004), 217-225. MR2041269

[Pan] P. Pansu. Introduction to $L^{2}$ Betti numbers. In Riemannian Geometry, pages 53-86. Amer. Math. Soc., 1996. MR1377309 (97c:58148)

[Rag] M. S. Raghunathan. Discrete Subgroups of Lie Groups. Springer-Verlag, 1972. MR0507234 $(58: 22394 \mathrm{a})$

[Re] R. Remak. Verallgemeinerung eines Minkowskischen Satzes. Math. Zeitschr. 17-18(1928), 1-34; 173-200.

[Sk1] B. F. Skubenko. A new variant of the proof of the inhomogeneous Minkowski conjecture for $n=5$ a. Trudy Mat. Inst. Steklov. 142(1976), 240-253, 271. MR.0563094 (58:27803)

[Sk2] B. F. Skubenko. A proof of Minkowski's conjecture on the product of $n$ linear inhomogeneous forms in $n$ variables for $n \leq 5$. J. Soviet Math. 6(1976), 627-650.

[So] C. Soulé. The cohomology of $\mathrm{SL}_{3}(Z)$. Topology 17(1978), 1-22. MR0470141 (57:9908)

[TW] G. Tomanov and B. Weiss. Closed orbits for actions of maximal tori on homogeneous spaces. Duke Math. J. 119(2003), 367-392. MR1997950 (2004g:22006)

[Va] P. Vámos. The missing axiom of matroid theory is lost forever. J. London Math. Soc. 18(1978), 403-408. MR0518224 (80a:05062)

[Wd] A. C. Woods. Covering six space with spheres. J. Number Theory 4(1972), 157-180. MR0302570 (46:1714)

Department of Mathematics, Harvard University, 1 Oxford St, Cambridge, MassaCHUSETTS 02138-2901 